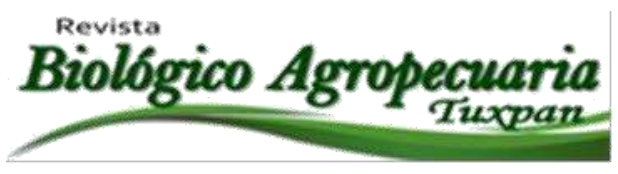

\title{
Adición de aceite de atún en dietas para pollos de engorda
}

Addition of tuna oil in diets for broiler chickens

\author{
Soni-Guillermo E. ${ }^{1 凶}$, Pérez-Sato M. ${ }^{1}$, Castro-González, N.P., García-Munguía A.M. ${ }^{2},{ }^{1}$ García-Munguía C.A. ${ }^{1}$, \\ Muñoz-Cuautle A. ${ }^{3}$ Cabrera-Cruz M.A. ${ }^{3}$ \\ ${ }^{1}$ Agronómica y Zootecnia, Facultad de Ingeniería Agrohidráulica, Benemérita Universidad Autónoma de \\ Puebla, Av. Universidad S/N, San Juan Acateno, Teziutlán Puebla. C.P. 73965 \\ ${ }^{2}$ Centro de Ciencias Agropecuarias, Universidad Autónoma de Aguascalientes, Av. Universidad, \#940, \\ Cd. Universitaria, Aguascalientes, Aguascalientes, C.P. $20131 .{ }^{3}$ Colegio de Posgraduados, Campus \\ Montecillos. Texcoco Estado de México \\ $\bowtie$ Autor para correspondencia:soniguillermo@hotmail.com
}

Recibido: 09/01/2016

Aceptado: 13/06/2016

\section{RESUMEN}

El objetivo del presente estudio fue evaluar el comportamiento productivo, características fisicoquímicas y sensoriales de la carne de pollo de engorde, alimentados con la inclusión de aceite de atún en la dieta. Los tratamientos fueron; T1: dieta testigo, T2: $2.5 \%$ de aceite de atún y T3: $3.5 \%$ de aceite de atún. Se utilizaron 90 pollos de la línea Roos, distribuidos en un diseño completamente al azar, con 30 repeticiones cada tratamiento, las variables evaluadas en comportamiento productivo en las etapas de iniciación y finalización fueron; consumo de alimento, ganancia de peso, conversión alimenticia; respecto a características de la carne fueron: $\mathrm{pH}$, rendimiento de la canal, capacidad de retención de agua y análisis sensorial. Los resultados mostraron diferencias $(\mathrm{p}<0.05)$ únicamente en ganancia de peso durante la etapa de finalización y sensorial de la carne. Se concluye que el $2.5 \%$ de inclusión de aceite de atún mejoró la ganancia de peso y sensorial de la carne, sin afectar las características fisicoquímicas y rendimiento de la carne.

Palabras clave: Aceite de atún, carne de pollo, alimentación de pollos.

\begin{abstract}
The aim of this study was to evaluate the productive behavior sensory, physicochemical characteristics and meat broiler, fed the inclusion of tuna oil in the diet. Treatments jurisdiction; T1: control diet, T2: $2.5 \%$ of tuna oil and $\mathrm{T}_{3} 3.5 \%$ of tuna oil. 90 chickens Roos line, distributed in a completely randomized design with 30 repetitions each treatment, the variables evaluated in productive behavior in the stages of initiation and completion were used; feed intake, weight gain, feed conversion; regarding meat characteristics were: $\mathrm{pH}$, carcass yield, water holding capacity and sensory analysis. The results showed differences $(\mathrm{p}<0.05)$ only in weight gain during the stage of completion. It is concluded that
\end{abstract}


the $2.5 \%$ inclusion of tuna oil improved weight gain and sensory meat without affecting the physicochemical characteristics and meat yield.

Key words: tuna oil, chicken meat, chicken feed

\section{INTRODUCCIÓN}

Las tendencias en el mercado de la carne indican un notable incremento del consumo de la carne de pollo en los últimos años, debido a su rápida producción y velocidad de crecimiento. Esta tendencia se atribuye, a las preferencias del consumidor y a los precios de los productos. Por mucho tiempo los aceites de pescado fueron un subproducto de la pesca destinado a usos industriales como la elaboración de margarinas de baja calidad y no se valoró su potencial como fuente de ácidos grasos $\Omega-3$ (Vásquez, 2002). Dada la relación entre nutrición y la salud humana como consecuencia de la creciente preocupación por la seguridad alimentaria, surge la posibilidad de utilizar el aceite de pescado en la dieta como vehículo para la ingestión de nutrimentos (ácidos grasos $\Omega-3$ ) y en la prevención y control de enfermedades (Castillo et al., 2005). Debido a los actuales hábitos de consumo, la ingestión de los ácidos grasos $\Omega-6$ ha aumentado al doble de los valores recomendados, alterando la relación de los ácidos grasos esenciales $\Omega-6: \Omega-3$, modificando la composición de los tejidos grasos, esto ha provocado un mayor consumo de aceites vegetales ricos en ácido linoleico $(\Omega 6)$ en relación a las fuentes marinas ricas en ácido linoleico $(\Omega 3)$, por lo que la relación $(\Omega 6 / \Omega 3)$ en muchos países es del orden de 15:1, cuando la relación ideal es 5:1 o máximo 10:1 (FAO, 1994). Para una buena salud humana se recomienda una relación óptima de ácidos grasos $\Omega-6$ : $\Omega-3$ de $6: 1$; sin embargo, el consumo de los ácidos grasos $\Omega-3$ ha disminuido hasta llegar a una sexta parte de lo requerido, esto debido a los cambios en el procesamiento de los alimentos (Castillo et al., 2005). Las modificaciones que se realizan a la alimentación animal con el propósito de obtener productos con nutrientes que sean benéficos a

los consumidores, implican cambios significativos en su composición química y propiedades físicas. Sin embargo, el uso de fuentes de $\Omega-3$ de origen marino, como el aceite de pescado, pueden generar olores y sabores desagradables, deteriorando la calidad de la carne (Tseng et al., 2000). Por tal motivo, el objetivo de este estudio fue evaluar el comportamiento productivo y sensorial en la carne de pollos de engorde alimentados con dietas con la inclusión de diferentes niveles de aceite de atún.

\section{MATERIALES Y MÉTODOS}

El experimento se realizó en el módulo avícola de la Facultad de Ingeniería

Agrohidráulica, Programa Ingeniería Agronómica y Zootecnia, de la Benemérita Universidad Autónoma de Puebla; ubicado en Tlatlauquitepec, Puebla en las coordenadas entre los paralelos $19^{\circ} 38^{\prime \prime}$ y $20^{\circ} 03^{\prime \prime}$ de latitud norte; meridianos $97^{\circ} 23^{\prime \prime}$ y $97^{\circ} 37^{\prime \prime}$ de longitud oeste y altitud de $1930 \mathrm{msnm}$. El clima que prevalece es templado húmedo con lluvias todo el año, con una precipitación media anual de $1264 \mathrm{~mm}$ y temperatura media anual de $15.1^{\circ}$ C (INEGI, 2009).

\section{Animales}

Se utilizaron 90 pollos de la línea Roos con peso inicial $100 \mathrm{~g}( \pm 0.005)$ de cinco días de nacidos. Los tratamientos fueron: $\mathrm{T}_{1} 0 \%$ de aceite de atún (testigo), T2 $(2.5 \%$ de aceite de atún) y $\mathrm{T}_{3}$ (3.5\% de aceite de atún). La duración del experimento fue de 56 días. 


\section{Alimentación}

Se elaboraron dos dietas (etapa de iniciación y finalización), estas fueron isoproteicas e isoenergéticas, formuladas a base de sorgo y pasta de soya (Cuadro 1 y 2) con la finalidad de cubrir los requerimientos de aminoácidos, energía, vitaminas y minerales para los pollos de engorde de acuerdo a los requerimientos sugeridos por el NRC (1996).

Cuadro 1. Dietas experimentales de iniciación utilizada para pollos de engorde con diferencia en niveles de aceite de atún 1-32 días.

\begin{tabular}{llll}
\hline Ingredientes & $\mathrm{T}_{1}=0 \%$ & $\mathrm{~T}_{2}=2.5 \%$ & $\mathrm{~T}_{3}=3.5 \%$ \\
\hline Sorgo & 60 & 60 & 60 \\
P. Soya & 33 & 33 & 33 \\
Aceite de atún & 0 & 2.5 & 3.5 \\
Aceite de soya & 3.5 & 1 & 0 \\
Metionina & 0.35 & 0.35 & 0.35 \\
Lisina & 0.2 & 0.2 & 0.2 \\
Treonina & 0.1 & 0.1 & 0.1 \\
Triptófano & 0.1 & 0.1 & 0.1 \\
CaCO3 & 1.4 & 1.4 & 1.4 \\
Ortofosfato de calcio & 1.35 & 1.35 & 1.35 \\
Prem. Vitaminas & 0.3 & 0.3 & 0.3 \\
Sal & 0.3 & 0.3 & 0.3 \\
Total & 100.0 & 100.0 & 100.0 \\
\hline & & Aporte de nutrientes & \\
\hline Proteína Cruda \% & 20.1 & 20.1 & 20.1 \\
EM (MCal/kg) & 2.9 & 2.9 & 2.9 \\
\hline
\end{tabular}

Las variables productivas a evaluar en las etapas de iniciación y finalización fueron: consumo de alimento, ganancia de peso y conversión alimenticia. El consumo de alimento y ganancia de peso se midieron semanalmente, una vez que se tuvieron estos valores se calculó la conversión alimenticia.
Para las variables de calidad de la canal, al concluir la engorda se sacrificaron diez pollos por cada tratamiento para medir rendimiento de la canal, $\mathrm{pH}$, capacidad de retención de agua y el análisis sensorial. El rendimiento de la canal se obtuvo dividiendo el peso caliente de la canal entre el peso vivo multiplicado por 100.

Cuadro 2. Dietas experimentales de finalización utilizada para pollos de engorde con diferentes niveles en aceite de atún 33-64 días.

\begin{tabular}{llll}
\hline Ingredientes & $\mathrm{T}_{1}=0 \%$ & $\mathrm{~T}_{2}=2.5 \%$ & $\mathrm{~T}_{3}=3.5 \%$ \\
\hline Sorgo & 69 & 69 & 69 \\
P. Soya & 24 & 24 & 24 \\
Aceite de atún & 0 & 2.5 & 3.5 \\
Aceite de soya & 3.5 & 1 & 0
\end{tabular}




\begin{tabular}{llll} 
Metionina & 0.15 & 0.15 & 0.15 \\
Lisina & 0.09 & 0.09 & 0.09 \\
Treonina & 0.1 & 0.1 & 0.1 \\
Triptófano & 0.4 & 0.4 & 0.4 \\
Carbonato de calcio & 1.5 & 1.5 & 1.5 \\
Ortofosfato de calcio & 0.67 & 0.67 & 0.67 \\
Prem. Vitamin & 0.25 & 0.25 & 0.25 \\
Sal & 0.35 & 0.35 & 0.35 \\
Total & 100.0 & 100.0 & 100.0 \\
\hline & & Aporte de nutrientes & 17 \\
\hline Proteína Cruda \% & 17 & 17 & 3.0 \\
EM (MCal/kg) & 3.0 & 3.0 & \\
\hline
\end{tabular}

El pH se midió al momento del sacrificio, a las 24 y 36 hrs potsmortem, la primera medición de $\mathrm{pH}$ se realizó directamente en el músculo Pectoralis major Agronómica y Zootecnia, Facultad de

Ingeniería Agrohidráulica, Benemérita Universidad Autónoma de Puebla, Av. Universidad S/N, San Juan Acateno, Teziutlán Puebla. C.P. 73965., posteriormente se pesaron $10 \mathrm{~g}$ de muestra a la que se le añadió $100 \mathrm{ml}$ de agua destilada para licuarse durante un minuto, se calibró el potenciómetro con soluciones buffer de 4 y 7 , se filtró la mezcla de carne en manta de cielo para eliminar el tejido conectivo y posteriormente se hizo la lectura del $\mathrm{pH}$, al término de cada medición se enjuagó el electrodo con agua destilada.

Para determinar la capacidad de retención de agua (CRA) se realizó 24 hrs postmortem, se pesaron 2 gramos de carne del músculo pectoralis finamente picada en un tubo de centrífuga, se homogeneizaron las muestras con $5 \mathrm{ml}$ de una solución $0.6 \mathrm{M}$ de cloruro de sodio y se agitaron en un vortex durante un min. Las muestras se dejaron reposar durante 30 min en un refrigerador a $4{ }^{\circ} \mathrm{C} \quad \mathrm{y}$ posteriormente se centrifugaron durante $15 \mathrm{~min}$ a 10,000 rpm en una centrifuga Beckman J-MI. El sobrenadante fue decantado y medido en una probeta. El volumen retenido de agua destilada se reporta como la cantidad de agua retenida en $100 \mathrm{~g}$ de carne (Guerrero, et al., 2002).

Para el análisis sensorial, se utilizó la prueba afectiva test de aceptación de acuerdo a la metodología descrita por Sánchez y William (2010) con el fin de determinar mediante un criterio personal subjetivo, si la muestra de carne presentada (pechuga de pollo) era aceptada o rechazada por la presencia de ligeros o fuertes sabores. Se sacrificaron 5 pollos por tratamiento; la carne se coció sin piel,

únicamente con agua (sin agregar condimentos). Se presentaron 80 muestras de $20 \mathrm{~g}$ por tratamiento de forma aleatoria; éstas fueron preparadas $\mathrm{y}$ presentadas de forma homogénea en forma de cubos. Esta prueba se llevó a cabo en el laboratorio de la Unidad Académica del Programa de Ingeniería Agronómica y Zootecnia de la BUAP, aplicando encuestas a 76 consumidores, se asignaron los valores de: sabor normal, ligeramente extraño y fuertemente extraño.

\section{Análisis estadístico}

Las variables de respuesta se analizaron con un diseño completamente al azar. Los datos se analizaron mediante modelo general lineal (GML) con el paquete estadístico SAS, 1998 y para la comparación de medias entre 
tratamientos se realizó con el procedimiento de Tukey (Steel y Torrie, 1988). Para el análisis sensorial se realizó la prueba no paramétrica de Kruskal-Wallis.

\section{RESULTADOS Y DISCUSIÓN}

El consumo de alimento no mostró diferencias significativas $(\mathrm{p} \geq 0.05)$ por efecto de los diferentes niveles de inclusión de aceite de atún en dietas para pollos de engorde en las atapas de iniciación y finalización tampoco en las semanas y en sus promedios. Sin embargo, el $\mathrm{T}_{2}(2.5 \%)$, presentó mayor consumo en ambas etapas, en comparación con $\mathrm{T}_{1}$ y $\mathrm{T}_{3}$. Castillo (2005), menciona que al adicionar niveles de 0,1 y $2 \%$ en dietas para gallinas de postura no encontraron diferencias significativas en esta variable; de igual manera Martínez (2008) con niveles de aceite de atún $0,0.75,1.5,2.25$ y $3.0 \%$ en una dieta basal maíz-pasta de soya, no encontraron diferencias significativas $(p>0.05)$ en ambas etapas para pollos en engorda. Estos resultados concuerdan con la presente investigación, a pesar de utilizar niveles mayores. Por otro lado Navidshad (2009), al adicionar niveles de aceite 0, 2 y 4\% evaluando el crecimiento y características de la canal de pollos de engorde, no encontró diferencias significativas a pesar de haber utilizado niveles superiores a los de la presente investigación.

En el Cuadro 3 se muestra la ganancia de peso en las diferentes etapas, se observaron diferencias significativas $(\mathrm{P} \leq 0.05)$ entre tratamientos adicionados con aceite de atún durante la semana cinco, esto se explica probablemente al cambio de la dieta de iniciación a finalización, sin embargo, en los promedios no se encontró efecto por los diferentes tratamientos, estos resultados concuerdan con Martínez (2008) quien sustituyó aceite de soya por aceite de atún ( 0 , $0.75, \quad 1.5, \quad 2.25$ y $3.0 \%$ ) encontrando diferencias significativas en la semana preliminar de la dieta de finalización. El nivel que mejoró la ganancia de peso fue el $\mathrm{T}_{2}(2.5 \%$ de aceite de atún) en comparación a $\mathrm{T}_{1}$ y T3; de igual manera Domínguez y Flores (2000) reportan ganancia de peso con el nivel máximo de $3 \%$ de aceite de atún. Al respecto Navidshad (2009) encontró que al evaluar diferentes niveles de aceite de pescado 0,2 y $4 \%$ en dietas para pollos de engorde, existen diferencias significativas $(\mathrm{p}<0.05)$ durante la etapa de finalización con un nivel de $2 \%$. Farhoomand y Checaniazer (2009) mencionan que la inclusión de aceite de pescado de $0,1,2$ y $3 \%$, la ganancia de peso se mejoró conforme se incrementaron los niveles de aceite. Mientras que Hulan et al. (1988) determinaron que al incrementar los niveles de aceite de pescado se disminuye la ganancia de peso, esto se lo atribuyeron a la palatabilidad del alimento.

En cuanto a la conversión alimenticia no se observaron diferencias significativas $(p \geq 0.05)$ por efecto de los diferentes tratamientos en las diferentes semanas y su promedio durante la fase experimental; sin embargo, los valores de conversión alimenticia disminuyeron conforme avanzaba el periodo semanal. Los resultados concuerdan con Castillo et al. (2005) quienes mencionan que al evaluar niveles de 0,1 y $2 \%$ de aceite de atún en dietas de pollo de engorda no se modificó la conversión alimenticia; de igual manera coincide con lo reportado por Navidshad (2009), quien no encontró diferencias significativas para esta variable al adicionar niveles de 0,2 y 4\% de aceite de pescado. Por el contrario Martínez (2008) al sustituir aceite de soya por aceite de atún con niveles $0,0.75,1.5,2.25$ y $3.0 \%$ no mostró diferencia significativa $(\mathrm{p} \leq 0.05)$ para esta variable.

Las variables de rendimiento de canal, $\mathrm{pH}$ y capacidad de retención de agua no presentaron diferencias significativas $(\mathrm{p} \geq 0.05)$ por efecto de la adición de aceite de atún $(0,2.5$ y $3.5 \%$ ) en esta investigación, los valores de rendimiento de canal coinciden con lo reportado por Navidshad (2009) quien al evaluar niveles de 0,2 y $4 \%$ de aceite de 
pescado, no encontró diferencias significativas para esta variable. Por el contrario, Farhoomand y Checaniazer (2009), indican que el nivel de aceite afecta el rendimiento de la canal con inclusiones de aceite de pescado de 0,2 y $4 \%$.

Los resultados obtenidos en la presente investigación fueron de 72, 68 y $66 \%$ para los $\mathrm{T}_{1}, \mathrm{~T}_{2}$ y $\mathrm{T}_{3}$ respectivamente; similares a los mencionados por Cuca (2009), quien obtuvo promedios que oscilan entre 66-68\%. La capacidad de retención de agua no se afectó al incrementar los niveles de aceite de atún, resultados similares a los de Liziac et al. (2013) en donde no encontró diferencias significativas por efecto de diferentes niveles de aceite de pescado en dietas para cerdos en finalización en pérdida por goteo, a pesar de ser de diferente especie.
A pesar de no haber diferencias significativas en el presente estudio los valores de $\mathrm{pH}$ se encuentran dentro de establecido en la literatura (Guerrero, 2009), por lo que se concluye que la carne satisfacía las características de frescura, ya que el $\mathrm{pH}$ es un indicador de la misma y su variación depende de la relación tiempo-temperatura postmortem, durante la cual se activan enzimas endógenas, prolifera la flora deteriorante y en consecuencia, se generan compuestos químicos que aumentan el $\mathrm{pH}$, un intervalo de $\mathrm{pH} 5.4$ a 5.8 en la carne post rigor indica que no se ha iniciado la producción de compuestos de putrefacción como aminas biogénicas, aldehídos, cetonas y ácidos grasos de cadena corta (Guerrero, 2009).

Cuadro 3. Ganancia de peso promedio (g animal-1) en pollos Ross en las etapas de iniciaciónfinalización alimentados con dietas adicionadas con aceite de atún.

\begin{tabular}{|c|c|c|c|c|c|}
\hline Etapas & Semana & T1 0\% & T2 $2.5 \%$ & T3 $3.5 \%$ & C.V.\% \\
\hline \multirow{4}{*}{ Iniciación } & 1 & $094.5^{\mathrm{a}}$ & $098.1^{\mathrm{a}}$ & $090.6^{\mathrm{a}}$ & 13.18 \\
\hline & 2 & $138.2^{\mathrm{a}}$ & $145.4^{\mathrm{a}}$ & $132.4^{\mathrm{a}}$ & 23.22 \\
\hline & 3 & $210.5^{\mathrm{a}}$ & $216.2^{\mathrm{a}}$ & $207.4^{\mathrm{a}}$ & 12.29 \\
\hline & 4 & $304.5^{\mathrm{a}}$ & $309.8^{\mathrm{a}}$ & $301.2^{\mathrm{a}}$ & 15.12 \\
\hline \multirow{4}{*}{ Finalización } & 5 & $393.7^{\mathrm{ab}}$ & $399.7^{a}$ & $388.7^{\mathrm{b}}$ & 25.12 \\
\hline & 6 & $436.7^{\mathrm{a}}$ & $442.5^{\mathrm{a}}$ & $428.3^{\mathrm{a}}$ & 12.92 \\
\hline & 7 & $578.5^{\mathrm{a}}$ & 586.7 & $571.0^{\mathrm{a}}$ & 14.31 \\
\hline & 8 & $687.3^{\mathrm{a}}$ & $695.7^{\mathrm{a}}$ & $684.8^{\mathrm{a}}$ & 18.68 \\
\hline \multicolumn{2}{|l|}{ Promedio } & $353.6^{\mathrm{a}}$ & $359.6^{\mathrm{a}}$ & $349.6^{\mathrm{a}}$ & 26.6 \\
\hline \multicolumn{3}{|c|}{$\begin{array}{l}\text { Los resultados del análisis sensorial } \\
\text { (Fig. 1) muestran que existen diferencias } \\
\text { significativas }(\mathrm{p} \geq 0.05) \text {, por efecto de los } \\
\text { diferentes tratamientos, y se observa que al } \\
\text { adicionar hasta } 3.5 \text { se detectaba el sabor de }\end{array}$} & \multicolumn{3}{|c|}{$\begin{array}{l}\text { concuerdan con Jeun et al. (2002) quienes } \\
\text { mencionan que la carne de pollos } \\
\text { alimentados con altos niveles de aceite de } \\
\text { pescado de } 3 \text { a } 3.5 \% \text { muestra un importante } \\
\text { incremento en el sabor a pescado. }\end{array}$} \\
\hline
\end{tabular}

aceite de atún en la carne. Estos resultados 


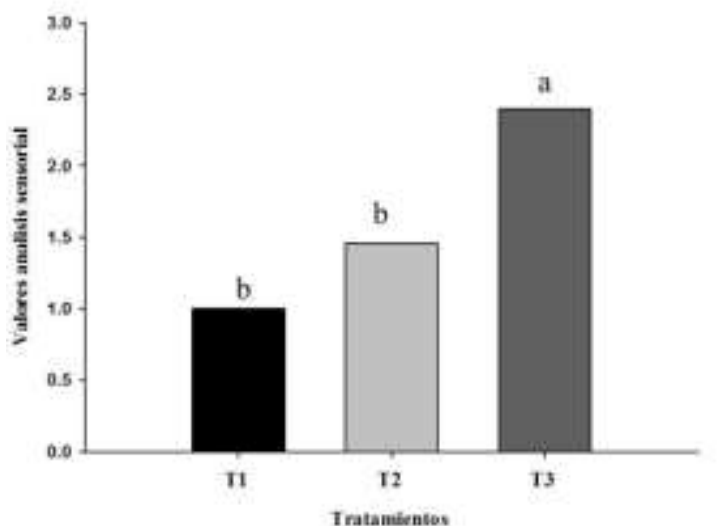

Figura 1. Análisis sensorial con la de aceptación de la carne de pollos Ross alimentados con dietas adicionadas con aceite atún. (a,b) Representan diferencias significativas entre tratamientos $(\mathrm{p} \leq 0.05)$

Este efecto puede deberse al desarrollo de un factor oxidativo más alto, derivando en una mala aceptación de la carne (Crawford et al., 1975), ya que un incremento de los ácidos $\Omega$-3 en las canales de los pollos significa una reducción en la estabilidad oxidativa (O’Keefe et al., 1995). Por otro lado López et al. (2001) utilizando niveles hasta de $4 \%$ de aceite de pescado no observaron efecto de rechazo de la carne por parte del consumidor, resultados diferentes a los del presente estudio.

\section{CONCLUSIÓN}

De acuerdo a los resultados encontrados en este trabajo se concluye que el porcentaje de inclusión de $2.5 \%$ de aceite atún nivel de aceite de atún mejora las variables productivas de los pollos de engorda y el análisis sensorial de la carne; sin embargo, las características fisicoquímicas como $\mathrm{pH}$ y capacidad de retención de agua no se modificaron por efecto de los diferentes tratamientos.

\section{LITERATURA CITADA}

Castillo-Badillo, J. L. Vázquez-Valladolid1, M. González-Alcorta , E. Morales-Barrera, R. M. Castillo-Domínguez y S. Carrillo-
Domínguez 2005. El aceite de atún como fuente de ácidos grasos w-3 en el huevo de gallina. Chapingo México. Grasas y Aceites. Vol. 56. 04 pp. https://doi.org/10.3989/gya.2005.v56.i2. Chekani A.S., Aghdam S.H., Maheri S.N., Ali R.A. and Vahdatpoor T. 2008. Omega-3 Fatty Acids Enrichment and Organoleptic Characteristics of Broiler Meat. Asian Journal of Animal y Veterinaria Avances. 3: 62-69. https://doi.org/10.3923/ajava.2008.62.69

Crawford, L., M. J. Kretsch, D. W. Peterson, and A. L. Lilyblade. 1975. The remedial and preventive effect of dietary alphatocopherol on the development of fish flavours in turkey meat. Journal of Food Science 40: 751755.https://doi.org/10.1111/j.1365-2621

Cuca G. M., 2009. Alimentación de aves. Departamento de Zootecnia. Universidad Autonoma de Chapingo 103-141 pp.

Domínguez G., L. y I. Flores G. 2000. Niveles óptimos biológico y económico de aceite de atún en dietas para pollo de engorda. Tesis Profesional. Universidad Autónoma Chapingo. Chapingo México. $58 \mathrm{pp}$.

FAO/OMS. 1994. Fat and oils in Human Nutrition. Report of a Join Expert Consultation FAO/OMS. FAO Food and Nutrition. No. 57. 
Farhoomand P. and Checaniazer S. 2009. Effects of graded levels of dietary fish oil on the yield and fatty acid composition of breast meat in broiler chickens. Faculty of Agriculture, Department of Animal Science. 159165. https://doi.org/10.3382/japr.2008-0

Guerrero, L. I., Ponce A. E, \& M. L. Pérez. (2002). Curso práctico de tecnología de carnes y pescado. Universidad Metropolitana Unidad Iztapalapa. D.F., México.

Guerrero, L.I. (2009). Meat spoilage detection. In: Nollet, L and F. Toldrá (eds). Handbook of processed meat and poultry analysis. CRC Press, Florida, E.U.A. 445-460. https://doi.org/10.1201/

Hulan H.W., Ackman R.G., Ratnayake W.M.N. and Proudfoot F.G. 1988. Omega-3 fatty acid levels and performance of broilers chickens fed redfish meal or redfish oil. Canadian Journal Animal Science. 68: 533-547. https://doi.org/10.

INEGI. 2009. Marco Geoestadistico municipal. Versión 3.1. Conjunto de datos de uso del suelo y vegetación.

Jeun- Horng, L., L. Yuan- Hui, and K. ChunChin. 2002. Effect of dietary fish oil on fatty acid composition, lipid oxidation and sensory property of chicken frankfurters during storage. Meat Science 60: 161-167. https://doi.org/10.1

Lisiak, D., E. Grzeskowiak, K. Borzuta, S. Raj, P. Janiszewski, and G. Skiba. 2013. Effects of supplementary vegetable and animal fats on the slaughter values of fatteners, meat quality, and fatty acid profile in pigs. Czech J. Anim. Sci. 58:497-511. https://doi.org/10.17221/704

Lopez-Ferrer, S., M. D. Baucells, A. C. Barroeta, and M. A. Grashorn. 2001. n3 enrichment of chicken meat. Use of very long- chain fatty acids in chicken diets and their influence on meat quality: fish oil. Poultry Science 80: 741-752.

https://doi.org/10.1093/ps/80.6.741
Martínez A., J. A. 2008. El aceite de atún como alternativa para enriquecer la carne de pollo con ácidos grasos Omega 3. Tesis de Maestría. Universidad Autónoma Chapingo. Texcoco, México. 12 pp.

Navidshad B., 2009. The Effects of Fish oil on growth performance and carcass characteristics of broiler chicks fed a low-protein diet. Journal Agric. Biol. 11: 635-638.

NRC, Nutrient Requirements of Poultry, 1996, National Research Council, 9a. ed. National Academic Press, Washington DC

O’Keefe, S. F., F. G. Proudfoot, and R. G. Ackman. 1995. Lipid oxidation in meats of omega- 3 fatty acids- enriched broiler chickens. Food Research International 28: 417- 424.https://doi.org/10.1016/096

Sanchez I.C. y William Albarracín. 2010. Analisis sensorial en carne. Facultad de ciencias agropecuarias. Universidad national de Colombia Pp. 227-239

SAS, (Statical Analisis Sistem).1998. SAS User's Guide Stadistics SAS INST.Inc. North Carolina USA. 1028 p.

Steel, R, G.D, y J.H. Torrie, 1988.Bioestadistica: Principios y Procedimientos. $\quad 2$ edicion. Edit.McGraw-Hill.Mexico, D.F. Pp

Tseng, Y. Y., C. J. Cheng, and R. C. Weng. 2000. Effects of dietary fish oil supplement on fatty acid composition and stability of pork meat and meat production. J. Anim. Sci. 13 (Suppl A): 126-129.

Vázquez V., J. 2002. Comportamiento productivo y de la calidad de huevo de gallinas alimentadas con aceite de atún en sustitución de aceite crudo de soya. Tesis de Maestría. Universidad Autónoma Chapingo. Texcoco México. $94 \mathrm{pp}$. 
Copyright (c) 2016 Soni-Guillermo E, Pérez-Sato M, Castro-González, N.P., Garcia-Munguia AM., G arcia-Munguia C.A, Murioz-Cuautle A. y Cabrera-Cruz M.A.

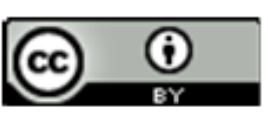

Este tex to está protegido por una licencia licencia CreativeCommons 4.0 .

Usted es libre para Compartir —copiar y redistribuir el m aterial en cualquier medio o formato- y Adaptar el documento — remezclar, transformar y crear a partir del material- para cualquier propósito, incluso para fines comerciales, siempre que cumpla la condición de:

Atribución: Usted debe dar crédito a la obra original de manera adecuada, proporcionar un enlace a la licencia, e in dicar si se han realizado cambios. Puede hacerlo en cualquier form a razonable, pero no de form a tal que sugiera que tiene el apoyo del licenciante o lo recibe por el uso que hace de la obra.

Resumendelicencia - Textocompletodelalicencia 